\title{
Sparfosic Acid
}

National Cancer Institute

\section{Source}

National Cancer Institute. Sparfosic Acid. NCI Thesaurus. Code C1398.

A stable transition state analogue for an aspartate transcarbamylase-catalyzed reaction with antineoplastic activity. Sparfosic acid is a stable transition analogue of the activated complex for the reaction catalyzed by aspartate transcarbamylase, the first step in the pyrimidine biosynthetic pathway. This agent inhibits de novo pyrimidine biosynthesis and increases the extent to which fluorouracil metabolites are incorporated into RNA. 